\title{
A new two-body relativistic potential model for pionic hydrogen
}

\author{
D.A. Kulikov*, R.S. Tutik ${ }^{\dagger}$ \\ Theoretical Physics Department, Dniepropetrovsk National University \\ 72 Gagarin av., Dniepropetrovsk 49010, Ukraine
}

\begin{abstract}
The new potential model for pionic hydrogen, constructed with the employment of the twobody relativistic equation, is offered. The relativistic equation, based on the extension of the $S L(2, C)$ group to the $S p(4, C)$ one, describes the effect of the proton spin and anomalous magnetic moment in accordance with the results of the quantum electrodynamics. Within this approach, using the experimental data on the strong energy level shift and width of the $1 s$ state in pionic hydrogen as input, the pion-nucleon scattering lengths have been evaluated to be $a_{\pi^{-} p}=0.0860(6) m_{\pi}^{-1}$ and $a_{\pi^{0} n}=-0.1223(19) m_{\pi}^{-1}$.
\end{abstract}

\section{Introduction}

One of the most important sources of information on strong interactions at low energy is the experiments performed with hadronic atoms [1]. In particular, the data on the shifts in energies and the widths in pionic $[2,3]$ and kaonic [4] hydrogen are used to determine the threshold parameters of the strong meson-nucleon S-matrix and the hadronic scattering lengths. These investigations have been carried out within the framework of the non-relativistic scattering theory [5-7], the effective field theory techniques [8-12] and the potential models [13-15].

The conventional potential model for pionic hydrogen, the bound $\pi^{-} p$ system, is the one-particle approximation based on the Klein-Gordon equation [13]. This model, however, does not provide a consistent description of some effects. So, the

\footnotetext{
*kulikov_d_a@yahoo.com

†tutik@dsu.dp.ua
} 
corrections raised by the proton spin and anomalous magnetic moment are added to the electromagnetic energies of the $\pi^{-} p$ system "by hand", using their values obtained in the quantum electrodynamics [16]. For the self-consistent description of these effects in pionic hydrogen to be possible it is needed to go beyond the one-particle approximation.

Up to now, the various approaches to deriving the relativistic two-body equations describing the fermion-boson systems have been offered [17-21]. Recently, the extension of the $S L(2, C)$ group to the $S p(4, C)$ one has been proposed for this purpose, too $[22,23]$. However, the last treatment permits us to deal not only with the ordinary Lorentz-scalar and Lorentz-vector potentials but also with the Lorentz-tensor one, being responsible for the interaction with anomalous magnetic moment.

The goal of the present work is to apply the above mentioned approach, based on the extension of the $S L(2, C)$ group, for constructing the model describing pionic hydrogen in the self-consistent manner within the framework of the relativistic twobody equation.

The outline of the Letter is as follows. In Section 2 we briefly consider the relativistic two-body equation based on the extension of the $S L(2, C)$ group and involving the Lorentz-scalar, Lorentz-vector and Lorentz-tensor potentials. In Section 3 the form of the potentials needed for the description of pionic hydrogen is specified. In Section 4 correctness of the corresponding two-body equation of the model is discussed. Section 5 deals with the electromagnetic binding energies for $\pi^{-} p$ system. Section 6 is devoted to the extraction of the hadronic scattering lengths from the data on the strong energy level shift and width of pionic hydrogen. Finally, conclusions are given in Section 7.

Throughout the Letter we use the Minkowski metrics $g^{m n}=\operatorname{diag}(1,-1,-1,-1)$ and the units in which $\hbar=c=1$.

\section{Two-body equation with the extension of the $S L(2, C)$ group}

It has been shown [22] that the extension of the $S L(2, C) \equiv S p(2, C)$ group to the $S p(4, C)$ one permits us to construct the wave equations for relativistic two-body systems. Keeping in mind the description of pionic hydrogen, we consider the sys- 
tem composed of a spin-1/2 fermion and a spin-0 boson. The wave function of the system is represented by a Dirac spinor or, in our treatment, by two $S p(4, C)$ Weyl spinors $\varphi$ and $\bar{\chi}$. The corresponding two-body wave equation without interaction is a straightforward generalization of the one-particle Dirac equation [23]

$$
P \bar{\chi}=\left(m_{+}+\tau^{1} \otimes I m_{-}\right) \varphi, \quad \tilde{P} \varphi=\left(m_{+}+\tau^{1} \otimes I m_{-}\right) \bar{\chi}
$$

where the $S p(4, C)$ momentum spin-tensor, $P$, and its conjugative, $\tilde{P}$, depend on the four-momenta of the constituent particles of the system through the quantities

$$
w_{m}=\frac{1}{2}\left(p_{1 m}+p_{2 m}\right), \quad p_{m}=\frac{1}{2}\left(p_{1 m}-p_{2 m}\right) .
$$

The mass parameters, $m_{ \pm}$, are related to the masses of the constituents by

$$
m_{+}=\frac{1}{2}\left(m_{1}+m_{2}\right), \quad m_{-}=\frac{1}{2}\left(m_{1}-m_{2}\right)
$$

whereas $I$ and $\tau^{i}$ stand for the unit $2 \times 2$ matrix and the Pauli matrices, respectively.

The above equation (1) must be supplemented with the subsidiary condition

$$
\left(w^{m} p_{m}-m_{+} m_{-}\right)\left(\begin{array}{c}
\varphi \\
\bar{\chi}
\end{array}\right) \equiv \frac{1}{4}\left(p_{1}^{2}-p_{2}^{2}-m_{1}^{2}+m_{2}^{2}\right)\left(\begin{array}{l}
\varphi \\
\bar{\chi}
\end{array}\right)=0,
$$

which guarantees that in the lack of the interaction the particles are on the mass shell, being subjected to the free Dirac and Klein-Gordon equations.

Because the wave equation (11) with the subsidiary condition (41) describes two systems, which differ from each other only in permutation of masses of the particles, we accept, for definiteness, that the Dirac fermion (proton) has the mass $m_{1}$ and the Klein-Gordon boson (pion) has the mass $m_{2}$.

As it has been shown [23], the introduction of the Lorentz-scalar, Lorentz-vector and Lorentz-tensor interaction potentials into the free wave equation (1) yields

$$
\begin{aligned}
& {\left[I \otimes \sigma^{m}\left(w_{m}+A_{m}\right)+\tau^{1} \otimes \sigma^{m}\left(p_{m}+B_{m}\right)\right] \bar{\chi}=\left[m_{+}+S_{+}+\right.} \\
& \left.\tau^{1} \otimes I\left(m_{-}+S_{-}\right)-\mathrm{i} I \otimes \sigma^{m} \tilde{\sigma}^{n} C_{m n}-\mathrm{i} \tau^{1} \otimes \sigma^{m} \tilde{\sigma}^{n} D_{m n}\right] \varphi \\
& {\left[I \otimes \tilde{\sigma}^{m}\left(w_{m}+A_{m}\right)+\tau^{1} \otimes \tilde{\sigma}^{m}\left(p_{m}+B_{m}\right)\right] \varphi=\left[m_{+}+S_{+}+\right.} \\
& \left.\tau^{1} \otimes I\left(m_{-}+S_{-}\right)-\mathrm{i} I \otimes \tilde{\sigma}^{m} \sigma^{n} C_{m n}-\mathrm{i} \tau^{1} \otimes \tilde{\sigma}^{m} \sigma^{n} D_{m n}\right] \bar{\chi}
\end{aligned}
$$

Here the spin-tensors $P$ and $\tilde{P}$ are rewritten in terms of the $2 \times 2$ matrices $\sigma^{m}=(I, \boldsymbol{\tau})$ and $\tilde{\sigma}^{m}=(I,-\boldsymbol{\tau})$ with $\boldsymbol{\tau}=\left(\tau^{1}, \tau^{2}, \tau^{3}\right)$, the Lorentz-scalar potentials, $S_{+}, S_{-}$, are 
involved through the mass substitutions whereas the Lorentz-vector potentials, $A_{m}$, $B_{m}$, and Lorentz-tensor ones, $C_{m n}, D_{m n}$, are involved through the minimal and nonminimal substitutions on the four-momenta, respectively.

Clearly, the obtained wave equation and the subsidiary condition must be compatible, i.e., the operators in their left-hand sides must commute. This can be achieved on assumption that the potentials must depend on the relative coordinate $x^{m}=x_{1}^{m}-x_{2}^{m}$ only through its transverse part

$$
x_{\perp}^{m}=\left(g^{m n}-w^{m} w^{n} / w^{2}\right) x_{n}
$$

with respect to the total four-momentum $w_{m}$, which is conserved and so can be treated as the eigenvalue rather than the operator.

Furthermore, the subsidiary condition (4) should be subject to the above substitutions on the masses and four-momenta, which should not change its form. All these result in the following restrictions on the shape of the potentials

$$
\begin{array}{ll}
\omega^{m} \pi_{m}+\pi_{m} \omega^{m}=2 w_{m} p^{m}, & M_{+} M_{-}+M_{-} M_{+}=2 m_{+} m_{-}, \\
C_{m k} D^{m n}+D_{m k} C^{m n}=0, & \omega^{m} D_{m n}-D_{m n} \omega^{m}+C_{m n} \pi^{m}-\pi^{m} C_{m n}=0
\end{array}
$$

where $\omega_{m}=w_{m}+A_{m}, \pi_{m}=p_{m}+B_{m}, M_{ \pm}=m_{ \pm}+S_{ \pm}$.

Once these conditions have been satisfied, the wave equation (5) supplemented with the subsidiary condition (44) provides a quantum description of the two-body problem, incorporating several important properties [23]. So, it is manifestly covariant, has correct one-particle limits and allows us to treat, in addition to the standard Lorentzscalar and Lorentz-vector potentials, also the interaction described by the Lorentztensor potentials.

\section{Specific form of potentials for pionic hydrogen}

Now let us specify the explicit form of the potentials needed for the description of pionic hydrogen. The restrictions on this form are as follows. Firstly, these potentials must obey the compatibility conditions (7). Secondly, the electromagnetic interaction between the proton and the pion should be described properly. This implies that the wave equation (5), being transformed into the semirelativistic Schrödinger-like form, should produce such relativistic kinetic energy, spin-orbit and Darwin terms which are 
consistent with the "improved Coulomb potential" of the quantum electrodynamics [16]. Thirdly, when the proton is assumed to be much heavier than the meson, Eq. (5) must be approximated by the Klein-Gordon equation with the ordinary scalar and vector potentials.

The Lorentz-vector potentials $A_{m}$ and $B_{m}$ satisfying the above restrictions can be chosen in the form

$$
A_{m}=(\mathcal{F}-1) w_{m}, \quad B_{m}=\left(\mathcal{F}^{-1}-1\right) p_{m}-\frac{\mathrm{i}}{2 \mathcal{F}^{2}} \frac{\partial \mathcal{F}}{\partial x_{\perp}^{m}}
$$

with $\mathcal{F}$ being a scalar function.

For the description of the electromagnetic interaction in pionic hydrogen to be correct, we accept the parametrization of $\mathcal{F}$ which follows the structure of the potential derived by summing the contributions of the relevant diagrams in the quantum electrodynamics [26]

$$
\mathcal{F}=(1-2 \mathcal{A} / E)^{1 / 2}
$$

where $E=2 \sqrt{w^{2}}$ denotes the total energy and $\mathcal{A}=\mathcal{A}\left(x_{\perp}^{2}\right)$ is the electrostatic (Coulomb) potential.

For the Lorentz-scalar potentials $S_{+}$and $S_{-}$, the simplest solution to the compatibility conditions (7) is given by

$$
S_{ \pm}=\frac{1}{2}\left(\left(m_{+}+m_{-}\right)^{2}+2 m_{w} \mathcal{S}+\mathcal{S}^{2}\right)^{1 / 2} \pm \frac{1}{2}\left(\left(m_{+}-m_{-}\right)^{2}+2 m_{w} \mathcal{S}+\mathcal{S}^{2}\right)^{1 / 2}-m_{ \pm}
$$

where

$$
m_{w}=m_{1} m_{2} / E \equiv\left(m_{+}^{2}-m_{-}^{2}\right) / E
$$

is the relativistic reduced mass, also referred to as the Todorov variable [24], and $\mathcal{S}=\mathcal{S}\left(x_{\perp}^{2}\right)$ is the scalar function, which goes over to the scalar potential of the Klein-Gordon equation in the limit when the proton is much heavier than the meson.

It should be added that the Lorentz-vector potentials can be chosen in either the electromagnetic-like form (8) or the time-like one. In the last case our Eq. (5) is transformed into the equation by Królikowski [17] (for details see Ref. [23]). However, for the point Coulomb interaction such time-like potentials do not lead to the correct relativistic recoil and Darwin terms in contrast to the electromagnetic-like Lorentzvector potentials [see Eq. (16) below]. 
Notice that all different momentum-independent forms of the Lorentz-scalar potentials, obeying the compatibility conditions, can be brought to the form (10) with the quantity $\mathcal{S}$ thought to be energy dependent. But for our purposes this energy dependence may be neglected because the binding energy of mesic hydrogen is small compared to the proton and meson masses.

In the following, only the manifestly covariant expressions (8) and (10) for the Lorentz-vector and Lorentz-scalar potentials will be used.

Concerning the Lorentz-tensor potentials $C_{m n}$ and $D_{m n}$, in order to satisfy the compatibility conditions (7), we must put $C_{m n}=0$. Due to the matrix contents of Eq. (5), the remaining potential $D_{m n}$ allows us to describe the interaction with the anomalous magnetic moment in the same form as the non-minimal coupling term introduced by Pauli [25] in the one-particle Dirac equation. By analogy with the Pauli term, we set

$$
D_{m n}=\frac{k_{1}}{4 m_{1}}\left(\frac{\partial A_{n}}{\partial x_{\perp}^{m}}-\frac{\partial A_{m}}{\partial x_{\perp}^{n}}+\frac{\partial B_{n}}{\partial x_{\perp}^{m}}-\frac{\partial B_{m}}{\partial x_{\perp}^{n}}\right),
$$

where $k_{1}$ and $m_{1}$ denote the anomalous magnetic moment and the mass of the fermion (for the proton, $k_{1}=1.793$ ).

\section{The two-body equation in the center-of-mass frame}

Although the wave equation (5) is relativistically invariant, it is convenient, for practical purposes, to pass to the center-of-mass frame. In this case we have $\mathbf{w} \equiv$ $\left(\mathbf{p}_{1}+\mathbf{p}_{2}\right) / 2=0, E=2 w_{0}$, so that the subsidiary condition (4) results in $p_{0}=$ $\left(m_{1}^{2}-m_{2}^{2}\right) /(2 E)$. Then Eqs. (4) and (5) are reduced to the Dirac-like form

$$
\begin{aligned}
& {\left[\boldsymbol{\tau} \cdot \mathbf{p}+\frac{\mathrm{i} k_{1}\left(E^{2} \mathcal{F}^{2}-m_{1}^{2}+m_{2}^{2}\right)}{4 m_{1} E^{2} \mathcal{F}^{2}} \boldsymbol{\tau} \cdot \boldsymbol{\nabla} \mathcal{A}\right] \phi} \\
& =\left[E_{1}-\mathcal{A}+M_{1} \mathcal{F}+\frac{k_{1}}{2 m_{1} E \mathcal{F}^{2}}(\boldsymbol{\tau} \cdot[\boldsymbol{\nabla} \mathcal{A} \times \mathbf{p}])\right] \psi, \\
& {\left[\boldsymbol{\tau} \cdot \mathbf{p}-\frac{\mathrm{i} k_{1}\left(E^{2} \mathcal{F}^{2}-m_{1}^{2}+m_{2}^{2}\right)}{4 m_{1} E^{2} \mathcal{F}^{2}} \boldsymbol{\tau} \cdot \boldsymbol{\nabla} \mathcal{A}\right] \psi} \\
& =\left[E_{1}-\mathcal{A}-M_{1} \mathcal{F}-\frac{k_{1}}{2 m_{1} E \mathcal{F}^{2}}(\boldsymbol{\tau} \cdot[\boldsymbol{\nabla} \mathcal{A} \times \mathbf{p}])\right] \phi,
\end{aligned}
$$

where $\phi=\mathcal{F}^{-1 / 2}\left(1+\tau^{1} \otimes I\right)(\bar{\chi}+\varphi)$ and $\psi=\mathcal{F}^{-1 / 2}\left(1+\tau^{1} \otimes I\right)(\bar{\chi}-\varphi)$ are the components of the Dirac bispinor, $M_{1}=\left(m_{1}^{2}+2 m_{w} \mathcal{S}+\mathcal{S}^{2}\right)^{1 / 2}, E_{1}=\left(E^{2}+m_{1}^{2}-m_{2}^{2}\right) /(2 E), m_{1}$ is the mass of the fermion and $m_{2}$ is the mass of the boson. 
Notice that, in the center-of-mass frame, the quantities $\mathcal{S}$ and $\mathcal{A}$ which determine all the involved potentials depend only on the distance between the particles, $\sqrt{-x_{\perp}^{2}}=|\mathbf{x}| \equiv\left|\mathbf{x}_{1}-\mathbf{x}_{2}\right|$. Therefore the spatial variables in Eq. (13) can be separated in the same manner as for the Dirac equation.

Thus, the derived equation (13) is assumed as a basis of our model for hadronic atoms. The correctness of its description of relativistic effects can be demonstrated with following limiting cases.

In the first case, it is supposed that the proton or, more likely, spin- $1 / 2$ nucleus is much heavier than the pion, $m_{1} / m_{2} \gg 1$. Then it is convenient to pass from the total energy $E$ to the energy variable by Todorov [24]:

$$
\epsilon_{w}=\left(E^{2}-m_{1}^{2}-m_{2}^{2}\right) /(2 E)
$$

which together with the relativistic reduced mass $m_{w}$ corresponds to the relative motion satisfying, in the lack of interaction, the effective one-body Einstein equation, $\epsilon_{w}^{2}-m_{w}^{2}=\mathbf{p}^{2}$.

Expanding all terms of Eq. (13) in powers of $1 / m_{1}$, eliminating the "small" component, $\psi$, in favor of the "large" one, $\phi$, and using the identity $\left(E_{1}-\mathcal{A}\right)^{2}-m_{1}^{2} \mathcal{F}^{2}=$ $\left(\epsilon_{w}-\mathcal{A}\right)^{2}-m_{w}^{2}$, Eq. (13) is reduced to the Klein-Gordon-like equation

$$
\left[\mathbf{p}^{2}+\left(m_{w}+\mathcal{S}\right)^{2}-\left(\epsilon_{w}-\mathcal{A}\right)^{2}\right] \phi=\frac{1}{m_{1}}\left[2 \mathcal{A}\left(2 m_{w} \mathcal{S}+\mathcal{S}^{2}\right)-\boldsymbol{\nabla} \mathcal{A} \cdot \mathbf{p}+(\boldsymbol{\tau} \cdot[\boldsymbol{\nabla} \mathcal{A} \times \mathbf{p}])\right] \phi
$$

that holds in the order of $1 / m_{1}$.

The left-hand side of this equation is the usual Klein-Gordon equation with the scalar and vector potentials, $\mathcal{S}$ and $\mathcal{A}$, in which the Todorov variables $m_{w}$ and $\epsilon_{w}$ appear instead of the reduced mass and energy, whereas the right-hand side does indeed contain the three corrections, raised by the heavy spin- $1 / 2$ particle, which describe the interference between the scalar and vector potentials, the Darwin term and the spin-orbit interaction, respectively.

The second limiting case is the semirelativistic approximation which is better suited for discussing the lowest-order relativistic corrections for pionic hydrogen. Now it is supposed that the meson mass is of the same order as the proton mass, but the binding energy, $\epsilon=E-m_{1}-m_{2}$, is much smaller than these masses, $\epsilon / m_{1,2} \ll 1$. Since the magnitudes of the potentials must also be small as compared to $m_{1,2}$, only the electrostatic potential, and not the strong interaction one, can be treated in this 
way.

Then, in the lack of the Lorentz-scalar (strong) interaction, after performing the expansion in powers of $1 / m_{1,2}$, Eq. (13) is transformed into

$$
\begin{aligned}
& \left(\frac{\mathbf{p}^{2}}{2 \mu}-\epsilon+\mathcal{A}\right) \phi=\left\{\frac{1}{2 \mu}\left[\left(1-\frac{3 \mu}{M}\right) \epsilon^{2}-2\left(1-\frac{\mu}{M}\right) \epsilon \mathcal{A}+\mathcal{A}^{2}\right]\right. \\
& \left.-\frac{1}{4 m_{1}^{2}}\left(k_{1}+\frac{1}{2}+\frac{m_{1}}{m_{2}}\right) \nabla^{2} \mathcal{A}-\frac{1}{2 m_{1} \mu}\left(k_{1}+\frac{1}{2}+\frac{m_{1}}{2 M}\right)(\boldsymbol{\tau} \cdot[\boldsymbol{\nabla} \mathcal{A} \times \mathbf{p}])\right\} \phi,
\end{aligned}
$$

where $M=m_{1}+m_{2}, \mu=m_{1} m_{2} / M$.

Putting $\mathcal{A}$ as the point Coulomb potential, we obtain just the same relativistic kinetic energy, spin-orbit and Darwin terms as those derived with the improved Coulomb potential' in the quantum electrodynamics [16].

Thus, we may conclude that the proposed relativistic two-body equation (13) with the electromagnetic interaction introduced through the Lorentz-vector and Lorentztensor potentials, (88) and (12), correctly describes the relativistic corrections for pionic hydrogen.

\section{$5 \quad$ Electromagnetic energies}

For evaluating the stationary electromagnetic binding energies of pionic hydrogen, we use the derived equation (13) without the strong interaction. As it has been shown, the electromagnetic interaction is introduced with the Lorentz-vector and Lorentztensor potentials. According to Eqs. (8) and (12), these potentials are determined by the single potential $\mathcal{A}$, which we represent as the sum of three parts

$$
\mathcal{A}_{\mathrm{e} . \mathrm{m} .}=\mathcal{A}_{\mathrm{pc}}+\mathcal{A}_{\mathrm{ext}}+\mathcal{A}_{\mathrm{vac}}
$$

Here $\mathcal{A}_{\mathrm{pc}}=-\alpha / r$ is the point Coulomb potential, $\alpha=1 / 137.036$;

$$
\mathcal{A}_{\text {ext }}=-\frac{\alpha}{r}(\operatorname{erf}(r / s)-1), \quad s^{2}=\frac{2}{3}\left(\left\langle r_{p}^{2}\right\rangle+\left\langle r_{\pi}^{2}\right\rangle\right)
$$

is the addition caused by the finite extension of the Gaussian charge distributions with $\left\langle r_{p}^{2}\right\rangle$ and $\left\langle r_{\pi}^{2}\right\rangle$ being the r.m.s. charge radii of the proton and pion;

$$
\mathcal{A}_{\mathrm{vac}}=\frac{\alpha}{\pi} \int_{0}^{1} \mathrm{~d} v \frac{v^{2}\left(1-v^{2} / 3\right)}{1-v^{2}}\left(\mathcal{A}_{\mathrm{pc}}(r)+\mathcal{A}_{\mathrm{ext}}(r)\right) e^{-2 m_{e} r / \sqrt{1-v^{2}}}
$$

is the Uehling potential, commonly used for describing the vacuum polarization at $O\left(\alpha^{2}\right)$ in hadronic atoms, which is smeared at short distances due to the finite extension of the charge distributions ( $m_{e}$ is the electron mass). 
Table 1: Contributions to the correction $\Delta \epsilon_{1 s}^{\mathrm{e} . \mathrm{m}}$. to the pionic hydrogen binding energy in eV. Numbers in brackets are the uncertainties due to uncertainties in the proton and pion charge radii.

\begin{tabular}{|l|l|l|l|l|}
\hline & This work & Ref. [13] & Ref. [9] & Ref. [21] \\
\hline Finite size effect & $0.106(3)$ & $0.102(3)$ & 0.100 & $0.102(9)$ \\
Vacuum polarization, order $O\left(\alpha^{2}\right)$ & -3.245 & -3.246 & -3.241 & \\
Relativistic recoil, proton spin and & & & & \\
anomalous magnetic moment & 0.047 & $0.047[16]$ & 0.047 & 0.0417 \\
Vacuum polarization, order $O\left(\alpha^{3}\right)$ & & -0.018 & & \\
Vertex correction & & 0.007 & & \\
\hline
\end{tabular}

Eqs. (13) with these potentials were solved numerically with the shooting method. For the pion charge radius, the value $\sqrt{\left\langle r_{\pi}^{2}\right\rangle}=0.663(6)$ fm deduced from the $\pi e$ scattering experiments [27] was used. The proton charge radius, $\sqrt{\left\langle r_{p}^{2}\right\rangle}=0.8750(68)$ $\mathrm{fm}$, and the masses of particles were taken from the PDG tables [28]. Using these values, the electromagnetic binding energy of the $1 s$ state in pionic hydrogen is found to be $\epsilon_{1 s}=-3238.252 \mathrm{eV}$ with an uncertainty of $0.002 \mathrm{eV}$ due to the uncertainties in the proton and pion charge radii.

In order to compare our result with those reported in literature, it is convenient to consider the difference between the electromagnetic binding energies obtained within the framework of our two-body description and the Klein-Gordon equation, which proved to be $\Delta \epsilon_{1 s}^{\text {e.m. }}=-3.092 \mathrm{eV}$. In fact, $\Delta \epsilon_{1 s}^{\mathrm{e} . \mathrm{m}}$. is the sum of the corrections due to the three different effects, namely, the vacuum polarization effect, the finite size effect and the effect of the relativistic recoil, the proton spin and the anomalous magnetic moment.

In Table 1, we compare the corrections to the electromagnetic binding energy of pionic hydrogen obtained with the present approach and with the other methods $[9,13,21]$.

It should be stressed that in Ref. [13] the finite size correction was evaluated within the Klein-Gordon equation by employing the Gaussian charge distribution, whereas the correction due to the relativistic recoil, the proton spin and the anomalous magnetic moment evaluated for the point Coulomb potential [16] has been added 
"by hand". In Ref. [9], the electromagnetic corrections were calculated within the framework of the chiral perturbation theory using the same input data as in Ref. [13]. Notice that nearly all the difference between our result and the results of these two approaches comes from the values of the r.m.s. proton and pion charge radii. In Ref. [21], the finite size effect and the relativistic correction were taken into account by employing the Breit-type equation. In contrast to Refs. $[9,16]$ as well as to our conclusion, the contribution of the proton anomalous magnetic moment in Ref. [21] is found to be negligible that slightly reduces the total relativistic correction, as seen from Table 1. This suppression seems to be caused by the sharp decrease of the hadron form factors, used in Ref. [21], at small $r$ (or at large momentum transfer), whereas the anomalous magnetic moment reveals itself only in this region.

Thus, we may conclude that the calculated corrections to the electromagnetic binding energy of pionic hydrogen are in good agreement with those quoted in literature. However, it should be stressed that, in contrast to the other approaches, the proposed relativistic two-body equation allows us to evaluate the finite size effect and the effect of the relativistic recoil, the proton spin and anomalous magnetic moment in the self-consistent way without resorting to the semirelativistic expansions in powers of $1 / c^{2}$. We anticipate that for heavier mesic atoms the developed approach may give the more distinguished values of the finite size corrections as compared to the other approaches, because the charge radii of the nuclei are larger.

\section{Pion-nucleon scattering lengths}

Now we are going to incorporate the strong interaction potential into the consideration and to calculate the pion-nucleon scattering lengths, using the existing experimental data on the strong energy-level shift and width of pionic hydrogen.

The extraction of the pion-nucleon scattering lengths will be done in the isospinsymmetrical single-channel approximation when the electromagnetic interaction is completely switched off and, by convention, the masses of the pions and nucleons are taken equal to the physical masses of the charged pion and proton, respectively. The detailed discussion of the influence of the isospin-breaking effects on the mesonnucleon scattering parameters can be found in Refs. [10,14]. 
Our relativistic model of pionic hydrogen utilizes the following potentials

$$
\mathcal{A}=\mathcal{A}_{\mathrm{e} . \mathrm{m} .}, \quad \mathcal{S}=U_{\text {str }},
$$

where it is assumed that the (optical) strong interaction potential $U_{\text {str }}$ is the Lorentzscalar and has the square-well form

$$
U_{\text {str }}(r)=\left\{\begin{array}{cl}
U_{0} & \text { for } r \leq \sqrt{\frac{5}{3}} r_{0} \\
0 & \text { otherwise }
\end{array}\right.
$$

For fitting the position and width of the quasistationary energy level of the $1 s$ state in pionic hydrogen, Eqs. (13) were solved numerically with varying the complex value of the strong interaction potential strength, $U_{0}$. Under realization of this procedure, the most recent experimental data by the PSI collaboration, $\epsilon_{1 s}^{\text {str }}=-7.120 \pm 0.008$ (stat) \pm 0.006 (syst) $\mathrm{eV}$ and $\Gamma_{1 s}=0.823 \pm 0.018 \mathrm{eV}$ [3], which are consistent with, but more precise than, the earlier ones [2], have been used. For adapting the experimental data to our single-channel approximation, the strong decay channel $\left(\pi^{-} p \rightarrow \pi^{0} n\right)$ was separated out with the replacement of the total decay width, $\Gamma_{1 s}$, by the partial width, $\Gamma_{1 s}^{\pi^{0} n}=\Gamma_{1 s} /\left(1+P^{-1}\right)$ where $P=1.546(9)$ is the Panofsky ratio [29].

After the potential strength, $U_{0}$, had been adjusted, the electromagnetic interaction was switched off and the radial equations were solved once again to produce the pion-nucleon scattering length [13]. The whole procedure was repeated with varying the parameter $r_{0}$ between $0.5 \mathrm{fm}$ and $1.5 \mathrm{fm}$.

The calculations with the exponential and Gaussian potentials instead of the square-well potential did not changed the values of $a^{\mathrm{h}}$, as it is expected for the effective-range theory.

The final results for the pion-nucleon scattering lengths in our models is

$$
a_{\pi^{-} p}=0.0860(6) m_{\pi}^{-1}, \quad a_{\pi^{0} n}=-0.1223(19) m_{\pi}^{-1} .
$$

It is to be pointed that the error in $a_{\pi^{-} p}$ arises mainly from the vague value of $r_{0}$ $\left(0.5 \mathrm{fm} \leq r_{0} \leq 1.5 \mathrm{fm}\right)$ and is reduced to $0.0002 m_{\pi}^{-1}$ if the value $r_{0}=1.0 \mathrm{fm}$ is fixed, whereas almost all the error in $a_{\pi^{0} n}$ comes from the experimental uncertainty in $\Gamma_{1 s}$. 


\section{Conclusion}

The main object of the present work was to construct the new potential model for describing pionic hydrogen within the framework of the relativistic two-body equation incorporating in the self-consistent manner the effects of the proton spin and anomalous magnetic moment.

With using the proposed model, the electromagnetic binding energy of pionic hydrogen has been evaluated without resorting to the semirelativistic expansions in powers of $1 / c^{2}$. This enabled us to treat non-perturbatively the finite extension of the charge distributions of the proton and meson. We have found that in the presence of the Gaussian charge distributions the corrections to the binding energy due to the relativistic recoil, the proton spin and the anomalous magnetic moment almost coincides with those obtained for the point Coulomb potential $[13,16]$. This is in contrast to Ref. [21], in which the contribution of the anomalous magnetic moment has proved to be negligible.

Furthermore, the pion-nucleon scattering lengths have been extracted from the experimental data on the strong energy level shift and width of the $1 s$ state in pionic hydrogen. It turns out that the effect of the proton spin and anomalous magnetic moment on the hadronic scattering lengths is exceedingly small. For instance, switching off the proton anomalous magnetic moment subtracts approximately $0.5 \times 10^{-4} m_{\pi}^{-1}$ from $a_{\pi^{-} p}$ that exceeds the precision of the experimental data. Notice that our results on the pion-nucleon scattering lengths are consistent with recent extractions within the framework of other potential models. In particular, values in Eqs. (22) agree reasonably well with quantities $a_{\pi^{-} p}=0.0859(6) m_{\pi}^{-1}$, $a_{\pi^{0} n}=-0.1243(15) m_{\pi}^{-1}$, calculated using the three-channel relativized potential model [14], and $a_{\pi^{-} p}=0.0870(5) m_{\pi}^{-1}, a_{\pi^{0} n}=-0.125(4) m_{\pi}^{-1}$, calculated using the non-relativistic scattering theory [7]. The agreement would be even better if we used, as in Refs. [7,14], the earlier value for the pionic hydrogen width [2] which is substantially larger than the latest one [3].

It is expected that the relativistic effects will be more profound for kaonic hydrogen because its properties are strongly influenced by the existence of the $\Lambda(1405)$ resonance. 


\section{Acknowledgments}

We thank Dr. J. Révai for discussing the details of his work [15]. This research was supported by a grant N 0106 U000782 from the Ministry of Education and Science of Ukraine which is gratefully acknowledged.

\section{References}

[1] C.J. Batty, E. Friedman, A. Gal, Phys. Rept. 287, 385 (1997).

[2] H.-Ch. Schröder et al., Eur. Phys. J. C 21 (2001) 473.

[3] L.M. Simons [Pionic Hydrogen Collaboration], Proceedings of International Workshop on Exotic Hadronic Atoms, Deeply Bound Kaonic Nuclear States and Antihydrogen: Present Results, Future Challenges, Trento, 2006, edited by C. Curceanu, A. Rusetsky and E. Widmann, p.8 arXiv:hep-ph/0610201.

[4] G. Beer et al., Phys. Rev. Lett. 94 (2005) 212302.

[5] S. Deser, M.L. Goldberger, K. Baumann, W. Thirring, Phys. Rev. 96 (1954) 774.

[6] T.L. Trueman, Nucl. Phys. 26 (1961) 57.

[7] T.E.O. Ericson, B. Loiseau, S. Wycech, Phys. Lett. B 594 (2004) 76.

[8] B.R. Holstein, Phys. Rev. D 60 (1999) 114030.

[9] V.E. Lyubovitskij, A. Rusetsky, Phys. Lett. B 494 (2000) 9.

[10] J. Gasser, M.A. Ivanov, E. Lipartia, M. Mojžiš, A. Rusetsky, Eur. Phys. J. C 26 (2002) 13.

[11] A.N. Ivanov, M. Faber, A. Hirtl, J. Marton, N.I. Troitskaya, Eur. Phys. J. A 18 (2003) 653.

[12] B. Borasoy, R. Nißler, W. Weise, Phys. Rev. Lett. 94 (2005) 213401.

[13] D. Sigg, A. Badertscher, P.F.A. Goudsmit, H.J. Leisi, G.C. Oades, Nucl. Phys. A 609 (1996) 310.

[14] G.C. Oades, G. Rasche, W.S. Woolcock, E. Matsinos, A. Gashi, Nucl. Phys. A 794 (2007) 73. 
[15] J. Révai, N.V. Schevchenko, Few Body Syst. 42 (2008) 83.

[16] G.J.M. Austen, J.J. de Swart, Phys. Rev. Lett. 50 (1983) 2039.

[17] W. Królikowski, Acta Phys. Pol. B 10 (1979) 739.

[18] H. Pilkuhn, J. Phys. B 25 (1992) 299.

[19] T. Tanaka, A. Suzuki, M. Kimura, Z. Phys. A 353 (1995) 79.

[20] S.N. Datta, A. Misra, J. Chem. Phys. 125 (2006) 084111.

[21] N.G. Kelkar, M. Nowakowski, Phys. Lett. B 651 (2007) 363.

[22] D.A. Kulikov, R.S. Tutik, A. P. Yaroshenko, Phys. Lett. B 644 (2007) 311.

[23] D.A. Kulikov, R.S. Tutik, Mod. Phys. Lett. A 23 (2008) 1829.

[24] I.T. Todorov, Phys. Rev. D 3 (1971) 2351.

[25] W. Pauli, Rev. Mod. Phys. 13 (1941) 203.

[26] H. Jallouli, H. Sazdjian, Phys. Lett. B 366 (1996) 409.

[27] S. Amendolia et al., Nucl. Phys. B 277 (1986) 168.

[28] C. Amsler et al., Phys. Lett. B 667 (2008) 1.

[29] J. Spuller et al., Phys. Lett. B 67 (1977) 479. 